\title{
Impact of two different commercial DNA extraction methods on BK virus viral load
}

\author{
Massimiliano Bergallo, ${ }^{1,2}$ Ilaria Galliano, ${ }^{1,2}$ Elisa Loiacono, ${ }^{3}$ Francesca Ferro, ${ }^{2}$ \\ Paola Montanari, ${ }^{1,2}$ Paolo Ravanini ${ }^{4}$ \\ ${ }^{1}$ Department of Public Health and Paediatric Sciences, University of Turin Medical School, Turin; \\ ${ }^{2}$ Laboratory of Citoimmunodiagnostics, University Hospital of City Science and Health, Regina \\ Margherita Children's Hospital, Turin; ${ }^{3}$ Nephrology, Dialysis and Transplantation, University \\ Hospital City of Science and Health, Regina Margherita Children's Hospital, Turin; ${ }^{4}$ Laboratory of \\ Molecular Virology, Azienda Ospedaliero-Universitaria Maggiore della Carità, Novara, Italy
}

\section{Summary}

Background and aim: BK virus, a member of human polyomavirus family, is a worldwide distributed virus characterized by a seroprevalence rate of 70-90\% in adult population. Monitoring of viral replication is made by evaluation of BK DNA by quantitative polymerase chain reaction. Many different methods can be applied for extraction of nucleic acid from several specimens. The aim of this studywas to assess the impact of two different DNA extraction procedure on BK viral load.

Correspondence: Massimiliano Bergallo, Department of Public Health and Pediatric Sciences, University of Turin, Medical School, 10136 Turin, Italy. Tel: +39.011 .3131652 .

E-mail: massimiliano.bergallo@unito.it

Key words: DNA extraction, BKV viral load, PCR real time, transplant recipients.

Acknowledgments: the authors thank the members of the Department of Public Health and Paediatrics for interesting discussions, and gratefully acknowledge the Director L. Cordero di Montezemolo.

Contributions: MB make substantial contributions to conception and design, IG and PM make substantial contributions to acquisition of data, EL and PM make substantial contributions to analysis and interpretation of data; MB and PR participate in drafting the article or revising it critically for important intellectual content; and MB give final approval of the version to be submitted and any revised version.

Conflict of interest: the authors declare no potential conflict of interest.

Received for publication: 11 November 2014.

Revision received: 19 January 2016.

Accepted for publication: 28 Janaury 2016.

(C) Copyright M. Bergallo et al., 2016

Licensee PAGEPress, Italy

Microbiologia Medica 2016; 31:4825

doi:10.4081/mm.2016.4825

This article is distributed under the terms of the Creative Commons Attribution Noncommercial License (by-nc 4.0) which permits any noncommercial use, distribution, and reproduction in any medium, provided the original author(s) and source are credited.
Materials and methods: DNA extraction procedure including the Nuclisens easyMAG platform (bioMerieux, Marcy l'Etoile, France) and manual QIAGEN extraction (QIAGEN Hilden, Germany). BK DNA quantification was performed by Real Time TaqMan PCR using a commercial kit.

Result and discussion: The samples capacity, cost and time spent were compared for both systems. In conclusion our results demonstrate that automated nucleic acid extraction method using Nuclisense easyMAG was superior to manual protocol (QIAGEN Blood Mini kit), for the extraction of BK virus from serum and urine specimens.

\section{Introduction}

BK virus, a member of human polyomavirus family, is a worldwide distributed virus characterized by a seroprevalence rate of $70-90 \%$ in adult population $(6,12)$.

BK virus establishes latency in uroepithelial cell, in B cell, brain, spleen and probably in other tissues. The virus can became reactivated in setting of immunodeficiency and result in cellular damage and organ dysfunction $(3,7,9,11)$.

Monitoring of viral replication is made by evaluation of BK DNA by quantitative polymerase chain reaction (PCR). The success and reliability of nucleic acid sequence amplification require efficient unbiased procedure of extraction $(2,15)$. A high-quality nucleic acid extract is expected to be free of amplification inhibitors and other substance that might affect enzyme substrates (10).

Many different methods can be applied for extraction of nucleic acid from several specimens. Common extraction procedures include phenol chloroform purification or the use of commercially available kits (5).

The aim of this study was to asses the impact of two different DNA extraction procedures including the Nuclisens easyMAG platform (bioMerieux, Marcy l'Etoile, France) and manual QIAGEN extraction, on viral load detection in serum and urine samples.

\section{Materials and Methods}

\section{Subjects}

A total of 52 specimens of which 28 urine and 24 serum samples were recruited by transplant patient afferent to Ospedale Maggiore 
della Carità (Novara, Northern Italy), twenty-four man and nine female with median age $57.18 \pm 12.8$ years. Ten sera and urine was obtained from healthy control subjects with median age $55.3 \pm 12.4$ referred to AVIS blood donor centre $S$. Anna hospital. Each healthy control completed a questionnaire to verify a good health status.

\section{Manual method (QIAGEN DNA blood MiniKit)}

The extraction procedure was performed with QIAGEN DNA blood MiniKit according to the manufacturer's instruction.

A maximum of 10 samples can be manually processed in each extraction.

Briefly: $20 \mu \mathrm{L}$ of protease and then $200 \mu \mathrm{L}$ of AL buffer were added to $200 \mu \mathrm{L}$ of each sample and extraction tubes were vortexed and incubated for $10^{\prime}$ at $56^{\circ} \mathrm{C}$. Two hundred $\mu \mathrm{L}$ of $96 \%$ ethanol was added and, the mixture was transferred to a QIAamp column and centrifuged for 1' at $6000 \mathrm{~g}$.

The column was put in a new collection tube, $500 \mu \mathrm{L}$ AW1 buffer was added and centrifuged for 1' at $6000 \mathrm{~g}$. This procedure was repeated with $500 \mu \mathrm{L}$ AW2 buffer. To remove all ethanol from the column it was put in a new collection tube and then subjected to a dry spin for 1' at full speed. Elution was performed by adding $50 \mu \mathrm{L}$ EL buffer for serum samples and $200 \mu \mathrm{L}$ for urine samples, incubating for 5 ' at room temperature following by centrifugation for 1' at $6000 \mathrm{~g}$.

\section{Automated method (easyMAG nuclisens extraction)}

Extraction with easyMAG was done according to the manufacture's recommendations. A maximum of 24 samples can be processed in each extraction. A total of $1000 \mu \mathrm{L}$ of urine and $500 \mu \mathrm{L}$ of serum samples were placed in the disposable sample vessel and then were loaded onto the extractor. After the initial lysis incubation, $100 \mu \mathrm{L}$ of magnetic silica, prepared as recommended by the manufacturer, was added to each sample, and the extractor was restarted.

Samples were eluted in $25 \mu \mathrm{L}$.

\section{Real-time PCR assays}

The quality of extracted DNA was tested in PCR amplification. BK DNA quantification was performed by Real Time TaqMan. PCR using a commercial kit (Elitechgroup, Milano Italy) with the 7300 Real Time PCR System (Applied Biosystems Monza, Italy). According to the manufacturer's instruction PCR amplification were set up in reaction volume of $25 \mu \mathrm{L}$ that contained $5 \mu \mathrm{L}$ of extracted sample, or negative control (sterile double-distilled $\mathrm{H}_{2} \mathrm{O}$ ) or standard plasmid dilutions $(100 ; 1000 ; 10000 ; 100000$ copies/ $\mu \mathrm{L})$.

Clinical specimens were processed with the following thermic profile: $50^{\circ} \mathrm{C}$ for $2 \mathrm{~min}$ (decontamination) and one cycle of initial denaturation at $95^{\circ} \mathrm{C}$ for $10 \mathrm{~min}$ followed by 45 cycles of $15 \mathrm{~s}$ at $95^{\circ} \mathrm{C}$ (denaturation), and $1 \mathrm{~min}$ at $60^{\circ} \mathrm{C}$ (annealing and extension).

Each sample was subjected to simultaneous TaqMan PCR for housekeeping gene human $\beta$-globin. Results were considered acceptable only in the presence of $\beta$-globin positivity. The results were classified as negative, high positive ( $>100,000$ copies/mL) and low positive $(<100,000$ copies/mL) or invalid if the internal control was not detected.

\section{Results and Discussion}

The aim of this study was to compare two different DNA extraction methods in order to establish their relative effectiveness for extracting viral DNA from serum and urine samples.

A total of 72 clinical specimens, which 38 urine and 34 serum samples, were used to comparative analysis of two different extraction procedures including manual QIAGEN extraction method and automated
Nuclisens easyMAG platform (bioMerieux, Marcy l'Etoile, France). According to the manufacturer's instruction, while manual extraction DNA kit used a silica gel membranes and enzymatic digestion with protease, the automated extraction procedure is based on Boom method. This is a mechanism by which DNA selectively binds onto glass particles (silica) in the presence of high concentration of chaotropic agent, such as guanidinium thiocyanate, while contaminant such as proteins, carbohydrates and ions do not, and subsequent washing and elution of nucleic acid (2).

After the extraction procedures were completed, the DNA was immediately quantified by Real Time Taq PCR using a commercial kit (Elitechgroup, Milano Italy).

Each samples was subjected to simultaneous TaqMan PCR for housekeeping gene human $\beta$-globin.

Results were considered acceptable only in presence of $\beta$-globin positivity.

The use of internal control could exclude false-negative results due to inhibitors present in clinical samples. For examples in blood samples the prosthetic group of haemoglobin released from erythrocytes following haemolysis, reversibly bind to Taq polymerase inhibiting its activity. Furthermore, there are different materials inhibit PCR by direct effect on Taq polymerase as follow: heparin, phenol, denatured albumin etc. (2). Urine has been found to be difficult specimens for PCR-based amplification, due to the presence of many possible inhibitors $(4,16)$.

The results were classified as negative, high positive and low positive or invalid if the internal control was not detected. No sample was invalid.

Overall, of 34 serum samples extracted with two different procedures, results were concordant for 30 samples and discordant for 4 , with a positive and negative results found by different methods for same samples. In particular, the results were concordant for positive samples with high titre of BK viral load and for negative samples. Considering discordant results, the Nanogen kit detected 20 samples extracted with automated method as low positive, in confront off only 16 samples extracted with manual method. The remainder 4 samples extracted with manual procedure were detected as negative.

Furthermore, considering 38 urine samples, the results were concordant for 28 samples extracted with both methods: 10 samples presents a high BK viral load, 10 specimens were negative and finally, 8 urine samples contain low BK viral load. The results were discordant for 10 urine samples. In particular, PCR Real Time was negative for 20 specimens extracted with Qiagen blood kit including 10 urine samples with low viral load, when the urine samples were extracted with Nuclisens easyMAG method (Table 1). The results were discordant for 4 serum samples. In particular, this samples resulted positive with low viral load when extracted with Nuclisens easyMAG method and negative when extracted with Qiagen blood kit (Table 2).

The differences in viral load between urine and serum samples extracted with manual and automated procedures respectively, could be explained by a operational complexity, defined as a number of manipulation required to obtain an extracted samples that was highest for QIAamp DNA Blood Mini kit (5). Most authors cite the

Table 1. Discordant results between urine samples extracted with automated method and manual procedure, tested in PCR amplification. BK DNA quantification was performed by Real Time TaqMan. PCR using a commercial kit (Elitechgroup, Milano Italy).

\begin{tabular}{lcccc} 
& $\begin{array}{c}\text { High } \\
\text { positive }\end{array}$ & $\begin{array}{c}\text { Low } \\
\text { positive }\end{array}$ & Negative & Total \\
Nuclisens easy MAG & 10 & 18 & 10 & 38 \\
QIAGEN Blood Mini kit & 10 & 8 & 20 & 38 \\
\hline
\end{tabular}


Table 2. Discordant results between urine samples (or serum samples) extracted with automated method and manual procedure, tested in PCR amplification. BK DNA quantification was performed by Real Time TaqMan. PCR using a commercial kit (Elitechgroup, Milano Italy).

\begin{tabular}{lcccc} 
& $\begin{array}{c}\text { High } \\
\text { positive }\end{array}$ & $\begin{array}{c}\text { Low } \\
\text { positive }\end{array}$ & Negative & Total \\
Nuclisens easy MAG & 4 & 20 & 10 & 34 \\
QIAGEN Blood Mini kit & 4 & 16 & 14 & 34 \\
\hline
\end{tabular}

Table 3. Comparison of cost, processing time, and additional materials required for the two extraction procedures.

\begin{tabular}{|c|c|c|}
\hline & $\begin{array}{l}\text { Nuclisens } \\
\text { easy MAG }\end{array}$ & $\begin{array}{c}\text { QIAGEN } \\
\text { Blood Mini kit }\end{array}$ \\
\hline Cost per extraction in Euro & 11 & 4.38 \\
\hline Approximate processing time & $50 \mathrm{~min}$ & $90 \mathrm{~min}$ \\
\hline Consumables/additional reagents & None & Tubes, ethanol \\
\hline
\end{tabular}

easyMAG as a user-friendly instrument, requiring little manipulation $(5,14)$. The number of manual specimens transfers was different in each methods. The manual procedure required three transfers, while the automated methods required two transfers, this results in a greater chance of error as nucleic acid loss, and the results would be a false negative. Furthermore, operational complexity in QIAamp DNA Blood Mini kit, may be cause false positive results due to cross contamination of negative specimens by strongly positive specimens.

These risks can contribute to incorrect decisions with potentially severe consequences for the patients. The evolution of BKV replication represents the basic strategy to early predict the onset of BKVAN, and to asses the clinical course thereof and monitor the response to treatment, too $(1,7,8,13)$.

The samples capacity, cost and time spent were compared for both systems (Table 3). The Nuclisens easyMAG, was able to process significantly more samples per run than QIAamp method. One to 24 samples can be analyzed in one run and DNA and RNA extraction can be performed within the same run, while a maximum of 10 samples can be manually processed in each extraction. The automated extraction method can be applied to a broad range of different specimens as blood, serum, urine etc.

The turnaround time for nucleic acid extraction with Nuclisens easyMAG was 50 min for 24 samples including 10 min of incubation with lysis buffer and about 10 min of hands-on time. Nucleic acid extraction of 10 samples with QIAGEN takes 90 min starting from addition of the enzyme.

The cost/sample of automated extraction method is higher than manual extraction procedure, as shown in Table 3.

\section{Conclusions}

In conclusion our results demonstrate that automated nucleic acid extraction method using Nuclisense easyMAG was superior to manual protocol (QIAGEN Blood Mini kit), for the extraction of BKV from serum and urine specimens, even though Nuclisense easyMAG was more expensive that manual extractor protocol.

\section{Reference}

1. Astegiano S, Bergallo M, Terlizzi ME, et al. Combined measurement of serum DNA and urine VP1 mRNA in monitoring BK virus replication in kidneys graft recipients. Transplant Proc 2011;43:1052-4.

2. Bergallo M, Costa C, Gribaudo G, et al. Evaluation of six methods for extraction and purification of viral DNA from urine and serum samples. New Microbiol 2006;29:111-9.

3. Dadhania D, Snopkowski C, Ding R, at al. Epidemiology of BK virus in renal-allograft recipients: indipendent risk factors for BK virus replication. Transplantation 2008;86:521-8.

4. Demmler GJ, Buffone GJ, Schimber CM, May RA. Detection of cytomegalovirus in urine from newborns by using polymerase chain reaction DNA amplification J Infect Dis 1988;158:1177-84.

5. Dundas N, Leos NK, Mitui M, et al. Comparison of automated nucleic acid axtraction methods with manual extraction. J Molecular Diagnostics 2008;10:311-6.

6. Hariharan S. BK virus nephritis after renal transplantation. Kidney Int 2006;69:655-62.

7. Hirsch HH, Brennan DC, Drachenberg CB, et al. Polyomavirusassociated nephropathy in renal transplantation: interdisciplinary analyses and recommendations. Transplantation 2005;79:1277-86.

8. Hirsch HH, Drachemberg CB, Steiger J, Ramos E. Polyomavirusassociated nephropaty in renal transplantation: critical issues of screening and management. Adv Exp Med Biol 2006;577:160-73.

9. Jin L, Jibson PE, Booth JC, et al. Genomic typing of BK virus in clinical specimens by direct sequencing of polymerase chain reaction products. J Med Virol 1993;41:11.

10. Leons K, Bergs K, Ursi D, et al. Evaluation of NucliSens EasyMAG for automated nucleic acid extraction from various clinical specimens. J Clin Microbiological 2007;45:421-5.

11. Nebuloni M, Tosani A, Boldorini R, et al. BK virus renal infection in a patient with the aquired immunodeficiency syndrome. Arch Pathol Lab Med 1999;123:807.

12. Nickeleit V, Singh HK, Mihatsch MJ. Polyomavirus nephropathy: morphology, pathophisiology, and clinuical menagment. Curr Opin Nephrol Hypertens 2003;12:599-605.

13. Ramos E, Drachemberg CB, Wali R, Hirsch HH. The decade of polyomavirus associated nephropathy: state of affairs. Transplantation 2009;87:621-30.

14. Tang YW, Sefers SE, Li H, et al. Comparison of five methods for extraction of Legionella pneumophila from respiratory specimens. J Clin Microbiol 2004;42:5913-6.

15. Wiedbrauk DL, Werner JC, Drevan AM. Inhibition of PCR by aqueous and vitreus fluid. J Clin Microbiological 1995;33:2645-6.

16. Yamaguchi Y, Hironoca T, Kajivara M, et al. Increased sensitivity for detection of human cytomegalovirus in urine by removal of inhibitors for polymerase chain reaction. J Virol Methods 1992;37: 209-18. 